\title{
Phenomenological scaling laws for "semidilute" macromolecule solutions from light scattering by optical probe particles ${ }^{a)}$
}

\author{
George D. J. Phillies, Gregory S. Ullmann, and Kathleen Ullmann \\ Department of Chemistry, University of Michigan, Ann Arbor Michigan 48109 \\ Thy-Hou Lin \\ Macromolecular Research Program, University of Michigan and Department of Chemistry, Louisiana State \\ University, Baton Rouge, Louisiana 70803
}

(Received 31 January 1985; accepted 18 February 1985)

\begin{abstract}
Polymer solution dynamics may be inferred from light scattering spectra of dissolved optical probe particles. We compare a variety of probes in solutions of several polymers. In the "overlapping" concentration/molecular weight regime, the Stokes-Einstein equation fails by up to a factor of 2, while the probe diffusion coefficient $D$ follows a scaling law $D$ / $D_{0}=\exp \left(-a M^{\gamma} c^{v} R^{\delta}\right)(c, M$, and $R$ are the polymer concentration, molecular weight, and the probe radius, respectively). Experimentally, $\gamma=0.8 \pm 0.1, v=0.6-1.0$, and $\delta=-0.1$ to 0 , contrary to the theoretical predictions $\gamma=0$ and $\delta=1$. With very high molecular-weight polymers, we observe a further "entangled" regime, characterized by huge $\left(10^{4}\right)$ failures of the Stokes-Einstein equation and the appearance of "fast" modes in the scattering spectrum.
\end{abstract}

\section{INTRODUCTION}

In the past decade there has been an increase in theoretical and fundamental experimental studies of macromolecule solutions. Much of this work has focused on the equilibrium and transport properties of polymers, either in the melt or after dilution with a small-molecule solvent. In particular, solutions have been extensively studied through static and dynamic light scattering from polymer concentration fluctuations. ${ }^{1}$ We here describe systematic results from the application of light scattering spectroscopy to ternary solvent:polymer:probe systems. Our method is physically analogous to the study of isotopically labeled polymer chains with neutron scattering, in that we observe the motion of a dilute macromolecule species through an unseen background. However, in contrast to many neutron scattering experiments, our "unlabeled" and "probe" species are chemically dissimilar.

In our work, the spectrum of the probe particles was isolated with an index-matching technique. With an isorefractive solvent:polymer pair, fluctuations in the polymer concentration scatter very little light. The scattered intensity is dominated by scattering from the probe particles. In the limit that the probe species is dilute (the dissolved polymer need not be dilute), we established a decade ago $^{2,3}$ that the initial slope of the spectrum $S(k, t)$ yields a diffusion coefficient $D$ which is indistinguishable from the single-particle diffusion coefficient of the probes. Probe motions are perturbed by the polymeric background; polymer dynamics can therefore be inferred indirectly from $D$.

Experiments on probe particles in a variety of systems (including protein solutions, ${ }^{4}$ water:polyethylene oxide, ${ }^{5}$ water:polyacrylic acid, ${ }^{6}$ and viscous small-molecule liquids $^{7}$ ) have previously been reported. Effects of solution

\footnotetext{
a) The support of this work by the National Science Foundation under Grant CHE82-13941 and by the National Institutes of Health under Grant GM31821 is gratefully acknowledged.
}

viscosity and temperature, probe radius $R$, and polymer composition, concentration $c$, and molecular weight $M$ were examined. Our purpose in this paper is interpretive. We seek to identify universal properties of solvent:polymer:probe mixtures by merging results from studies on many different systems. These phenomenological conclusions are given in Sec. II. There are also a series of hydrodynamic calculations on the diffusion of spheres in polymer solutions and gels; these are summarized and tested against experiment in Sec. III. Section IV contains a discussion.

\section{PHENOMENOLOGY}

Our experimental methods and detailed findings have already been published. ${ }^{4-7}$ Our light scattering spectrometers employ a $20 \mathrm{~mW}$ He-Ne laser, constant $90^{\circ}$ scattering angle, 64 or 144 channel digital correlator, and data analysis with the method of cumulants. Since the observed diffusion coefficients were larger than expected, extensive precautions were taken to ensure that we had not overlooked a relaxation on a time scale longer than the one being studied intensely. Polymers studied were polyethylene oxide (PEO) (molecular weights $7500,18000,1 \times 10^{5}$, and $3 \times 10^{5}$ ), polyacrylic acid (PAA) (molecular weights $50000,3 \times 10^{5}$, and $\left.1 \times 10^{6}\right)$, and bovine serum albumin (BSA), all in aqueous solution. The probe particles were carboxylate-modified polystyrene latex (PSL) spheres (radii included $208 \AA, 517 \AA$, $800 \AA, 0.32 \mu$, and $0.62 \mu)$ and BSA. The probes and polymers are commercial preparations, which were not refractionated. The scattering cross sections of PAA, PEO, BSA, and PSL are such that BSA may either be used as a probe (in PEO or PAA solutions) or as a nearly non-scattering background (relative to PSL as a probe). The PSL concentration was sufficiently low ( $<0.01 \%$ by weight) that polymer adsorption by the PSL could not have changed the polymer concentration appreciably. To avoid electrostatic artifacts, BSA:PEO and PSL:BSA solutions also contained $0.5 \mathrm{M}$ $\mathrm{NaCl}$ and 0.1 M HEPES buffer, $p \mathrm{H}$ 7. PAA solutions had no 
added salt or base. As will be reported elsewhere, we attempted to use lysozyme as a nondilute probe of PEO solutions. $^{8}$ These studies are not relevant here because the reported $^{9}$ water:lysozyme critical point is actually a cryoprecipitation (salting-out) effect. Table I indicates two dozen combinations of probe radii, polymer molecular weights, and polymer concentrations which we have studied effectively.

We find that phenomenology and polymer molecular weight divide our systems into four regimes, as described in detail below. Solutions of low molecular weight polymers behave like small-molecule liquids. With increasing molecular weight there is some evidence for transition effects, but most of our data fall into a third "overlapping" regime. Concentrated solutions of the largest polymer studied $\left(1 \times 10^{6}\right.$ amu PAA) show a qualitatively different "entangled" behavior. Our overlapping and entangled regimes are not equivalent to the "semidilute" and "concentrated" regimes of the French school, in that the occurrence of our regimes depends on the polymer molecular weight, while for a given long-chain polymer one can move from the semidilute to the concentrated regime purely by increasing the concentration.

In solutions of low molecular weight polymers $(75000$ amu PEO, 50000 amu PAA), $D$ follows the Stokes-Einstein equation:

$$
D=\frac{k_{B} T}{6 \pi \eta R},
$$

$R$ being the nominal probe radius and $\eta$ the macroscopic shear viscosity. $\eta$ was obtained with calibrated Ubbelohde and Cannon-Fenske (capillary) viscometers. The viscosity depends substantially on the chemical nature, molecular

TABLE I. Fits of experimental measurements of the diffusion coefficient to Eq. (2) for various polymers and probe species. (PEO $=$ polyethylene oxide:water; PAA = polyacrylic acid:water; $\mathrm{PSL}=$ polystyrene latex; BSA= bovine serum albumin.)

\begin{tabular}{|c|c|c|c|c|c|c|}
\hline Polymer & $M$ & $c$ & Probe & $R$ & $\alpha$ & $v$ \\
\hline \multirow[t]{13}{*}{ PEO } & 7500 & $0-350$ & PSL & $208 \AA$ & 0.035 & 0.81 \\
\hline & & & & $517 \AA$ & 0.034 & 0.83 \\
\hline & & & & $0.32 \mu$ & 0.036 & 0.82 \\
\hline & $18500^{b}$ & $0-50$ & PSL & $208 \AA$ & 0.076 & 0.78 \\
\hline & & & & $517 \AA$ & 0.061 & 0.84 \\
\hline & $1 \times 10^{5 b}$ & $0-3$ & PSL & $208 \AA$ & $(0.013)^{\mathrm{a}}$ & $(1.95)$ \\
\hline & & & & $517 \AA$ & 0.046 & 1.35 \\
\hline & & & & $0.32 \mu$ & 0.064 & 1.00 \\
\hline & $3 \times 10^{5 b}$ & $0-3$ & PSL & $208 \AA$ & 0.14 & 0.88 \\
\hline & & & & $517 \AA$ & 0.14 & 0.92 \\
\hline & & & & $0.32 \mu$ & 0.12 & 0.82 \\
\hline & $1 \times 10^{5 b}$ & $0-5$ & BSA & $37 \AA$ & 0.08 & 0.6 \\
\hline & $3 \times 10^{5 b}$ & $0-3$ & BSA & $37 \AA ̊$ & 0.25 & 0.76 \\
\hline \multirow[t]{9}{*}{ PAA } & $5 \times 10^{4}$ & $0-150$ & PSL & $204 \AA$ & 0.047 & 0.79 \\
\hline & & & & $800 \AA$ & 0.029 & 0.88 \\
\hline & $3 \times 10^{5 b}$ & $0-150$ & PSL & $204 \AA$ & 0.236 & 0.65 \\
\hline & & & & $800 \AA$ & 0.208 & 0.66 \\
\hline & & & & $0.62 \mu$ & 0.184 & 0.67 \\
\hline & & & & $1.5 \mu$ & 0.151 & 0.67 \\
\hline & $1 \times 10^{6 b}$ & $0-20$ & PSL & $204 \AA$ & 0.227 & 0.63 \\
\hline & & & & $800 \AA$ & 0.262 & 0.54 \\
\hline & & & & $0.62 \mu$ & 0.696 & 0.74 \\
\hline \multirow[t]{3}{*}{ BSA } & {$[67000]^{b}$} & $0-200$ & PSL & $517 \AA$ & 0.0080 & 0.92 \\
\hline & & & & $0.32 \mu$ & 0.0053 & 0.97 \\
\hline & & & & $0.62 \mu$ & 0.0044 & 0.96 \\
\hline
\end{tabular}

${ }^{a}()=$ marginal results.

${ }^{b}$ Stokes-Einstein equation fails. weight, and concentration of the polymer; viscosities from 0.9 to $>1000 \mathrm{cP}$ were encountered.

In 18000 amu PEO, we observe an anomaly; the diffusion coefficients of $517 \AA$ and $0.62 \mu$ PSL increase with increasing polymer concentration. The increase is reproducible, and substantially larger than the experimental error. The $517 \AA$ probe particles show a similar behavior in moderately concentrated $(20-100 \mathrm{~g} / \mathrm{L}) \mathrm{BSA}$ solutions. $D / D_{0}$ becomes as large as 1.5. D would increase if the polymer acted to disperse aggregated spheres. For dispersal to cause the observed increase in $D$, many large aggregates would need to be present before the polymer was added. However, the apparent radius of the spheres in plain water is in reasonable agreement with the manufacturer's nominal sphere radius (based on electron microscopy and other techniques) suggesting that dispersable (or other) aggregates are not initially present in large amounts. We therefore do not interpret the anomalous positive slope of $d D / d c$ as arising from particle dispersion. In the absence of further examples, a detailed interpretation of the phenomenon seems premature.

Probe particles in solutions of BSA or large synthetic polymers do not obey the Stokes-Einstein equation. In most of these systems, $D$ falls with increasing $c$, but $\eta$ depends on $c$ more strongly than $D$ does, so that $D$ is larger (by as much as a factor of 2) than predicted by Eq. (1). Failures of the Stokes-Einstein equation increase with increasing polymer concentration, and are at least as apparent for large spheres as for smaller spheres. For PSL in BSA or $3 \times 10^{5}$ amu PAA, the failure clearly increases with increasing probe size. We identify systems showing factor of 2 level failures of the Stokes-Einstein equation as being in the overlapping regime.

Polymer adsorption by the probes, or polymer-driven protein aggregation, ${ }^{10}$ would increase $R$, thereby reducing $D$ relative to Eq. (1); polymer adsorption/aggregation thus has the wrong sign to explain the behavior of $D$ in the overlapping regime. Indeed, in PSL:PEO we were able to use nonabsorbing probes to quantitate the failure of Eq. (1), thereby allowing us to measure the (hydrodynamic) thickness of a PEO layer absorbed onto PSL. 5

In the overlapping regime $D$ is represented well by

$$
D / D_{0}=e^{-\alpha c^{2}},
$$

$D_{0}$ being $D$ in pure water, and $\alpha$ and $v$ being adjustable parameters. Table I gives measurements of $\alpha$ and $v, v$ depends primarily on the polymer species, not on $M$ or $R$, while $\alpha$ varies substantially from system to system. Figure 1 plots $\alpha$ against $M$. To account for the structural difference between BSA (a globular protein with little enclosed water) and PEO (a loosely coiled polymer whose hydrodynamic radius encompasses much solvent), BSA points were assigned an effective molecular weight $M_{e}$ chosen so that a polyethylene oxide species with $M=M_{e}$ would have a radius of gyration equal to the $37 \AA$ hydrodynamic radius of BSA. (Equivalently, the abscissa of Fig. 1, while labeled as $M$, is effectively the radius of gyration.) A least-squares fit shows $\alpha \sim M^{\gamma}$ with $\gamma=0.8 \pm 0.1$. The dependence of $\alpha$ on $R$ is weak, and hence difficult to obtain. The best value for the dependence, because the data cover the widest range of $c$, is from PSL in $3 \times 10^{5}$ amu PAA, for which $\alpha \sim R^{\delta}$ with $\delta=-0.1 .^{6}$ 


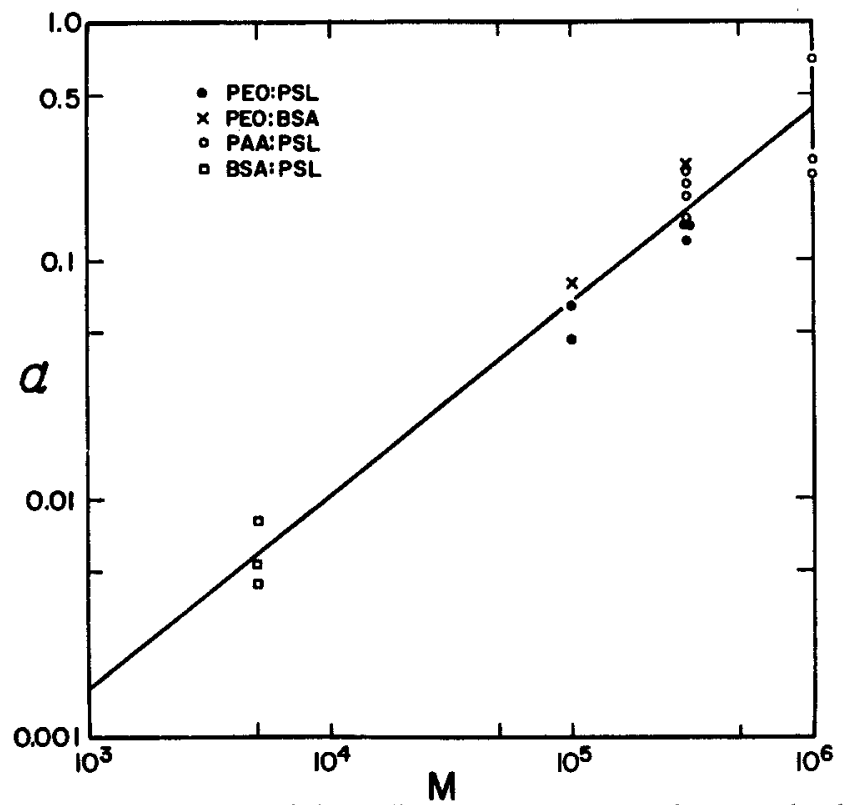

FIG. 1. Dependence of the scaling parameter $\alpha$ on polymer molecular weight $M$, using the data of Table I for systems in which Eq. (1) fails. Solid line: best fit of $\alpha \sim M^{\gamma}$ gives $\gamma=0.8 \pm 0.1$. Note that the PEO:BSA points are above the PEO:PSL points; small probes are retarded more than large ones.

The full scaling equation implicit in Eq. (2) is

$$
D / D_{0}=e^{-a c^{2} R^{s} M^{r}},
$$

where from our data $v \cong 0.6-1.0, \gamma=0.8 \pm 0.1$, and $\delta=0$ to -0.1 .

Solutions of $1 \times 10^{6}$ amu PAA show qualitative features which are not apparent in solutions of lower molecularweight polymers. From these features, we infer the appearance of a new "entangled" regime in concentrated solutions of very long polymers. Solutions of the smaller polymers satisfy $\eta / \eta_{0}=\exp \left(a c^{v}\right)$, but for $1 \times 10^{6}$ amu PAA a $\log -\log$ plot of $\eta$ against $c$ shows a straight line for $c>2 \mathrm{~g} / \mathrm{L}$, i.e., ${ }^{4}$

$$
\eta / \eta_{0}=a c^{3.15} \text {. }
$$

The 3.15 power of Eq. (2) should not be confused with the well-known $M^{3.4}$ power law dependence of $\eta$ in entangled polymer solutions. Furthermore, in this regime, at concentrations above $2 \mathrm{~g} / \mathrm{L}$, the spectral second cumulant $v$ shows a substantial increase. A more detailed examination reveals that the increase in $v$ is caused by the appearance of a new "fast" spectral mode, whose decay rate is an order of magnitude larger than that of the mode which we ${ }^{6}$ used to compute $D$. Similar modes appear to be present for PSL in 750000 amu PAA. The dependence of $D$ on $c$ and $R$ is stronger in the entangled regime than in the overlapping regime. In the entangled regime, the Stokes-Einstein equation fails by as much as four orders of magnitude, $D$ being far larger than expected from $\eta$. Finally, in this regime $D / D_{0}$ or large $(0.62$ $\mu$ ) spheres is as much as an order of magnitude smaller than $D / D_{0}$ of small $(204,800 \AA)$ spheres, contrary to the finding in the overlapping regime that $D / D_{0}$ and $\alpha$ are nearly independent of $R$.

\section{THEORY OF SPHERE: POLYMER MIXTURES}

The diffusion of a Brownian sphere through a longchain polymer solution or gel has been treated theoretically by several authors, including Ogston et al., ${ }^{11}$ Cukier, ${ }^{12} \mathrm{Al}-$ tenberger and Tirrell, ${ }^{13}$ and Langevin and Rondelez. ${ }^{14} \mathrm{De}-$ spite using very different physical models, these calculations predict similar forms for the concentration dependence of $D$.

Ogston et al. " modeled a gel as a suspension of long rigid rods, though which a sphere passed by Brownian motion. There were no hydrodynamic interactions between the sphere and the rods. In order to be able to move, a sphere had to fit through the gaps between neighboring rods. For randomly stacked rigid rods, Ogston obtained the distribution function of gap sizes by direct statistical analysis. Larger spheres were less likely to be confronted with holes through which they could pass, so that

$$
D / D_{0}=e^{-\kappa R} \text {, }
$$

where $D_{0}$ is the probe diffusion coefficient in pure solvent and $\kappa$ is a scaling constant; $\kappa \sim c^{1 / 2}$. In this model, large spheres are restrained more than small ones are. Ogston's theory does not include a percolation analysis. The fact that a sphere is faced with a hole, through which it can fit, does not mean that the sphere is in a tube (a series of holes) which is large enough to let the sphere pass from one end of the solution to the other. Ogston's theory is therefore more appropriate for motion over short distances than over long distances.

Cukier ${ }^{12}$ modeled a polymer solution as a hydrodynamic medium in which the usual Navier-Stokes equation is replaced by a screened Navier-Stokes equation

$$
\eta \nabla^{2} \mathbf{V}(\mathbf{r})-\nabla P(r)=\mathbf{F}(\mathbf{r})+\kappa^{2} \mathbf{V}(\mathbf{r})
$$

Here $\eta$ is the shear viscosity of the fluid, $V(r)$ is the fluid's velocity field, $P(\mathbf{r})$ is the local pressure, $F(\mathbf{r})$ is the external force, and $\kappa$ is a screening length. The screening was assumed to arise from the interaction of the fluid with a background suspension of externally fixed frictional centers, the frictional centers modeling the monomer beads on rod-like or coiled polymer chains. The drag coefficient was obtained with the usual derivation of Stokes' Law, except that Eq. (5) replaced the familiar, unscreened, Navier-Stokes equation. True collisions (nonhydrodynamic interactions) between the sphere and the polymer chains were not included in the model. Cukier found

$$
f=f_{0}(1+\kappa R) \cong f_{0} \exp (\kappa R),
$$

where $f=k_{B} T / D$ is the drag coefficient, the subscript 0 represents the absence of added polymer, and the final equality stems from an effective-medium approximation. $\kappa$ depends on the polymer concentration. deGennes' assertion ${ }^{15}$ that $\kappa$ and the polymer scaling length $\xi$ have the same dependence on concentration implies $\kappa \sim c^{v}$ for $v$ in the range $0.5-1.0$.

Altenberger and Tirrell ${ }^{13}$ treat diffusion in a polymer gel as a scattering problem in which the moving particle sets up a wake which bounces off a suspension of fixed spheres, the fixed spheres representing the gel. The rescattered wake eventually acts back on its source, retarding diffusion. The bare propagator for the wake was the Oseen tensor. Because the Oseen tensor is long range, the dependence of $D$ on the sphere concentration is nonanalytic. A resummation leads to an exponentially screened Oseen tensor. Altenberger and Tirrell predict 


$$
D / D_{0}=1-a c^{1 / 2}-b c^{1} .
$$

$a$ and $b$ are constants given explicitly in Ref. 13; $a$ is linear in $R$.

Langevin and Rondelez $z^{14}$ present physical arguments about probe diffusion based on deGennes' ideas on semidilute polymer solutions. Very large probes were assumed to see the solution as a continuum, for which the Stokes-Einstein equation was assumed to hold. Very small probe particles were assumed to respond only to the solvent viscosity $\eta_{0}$. The only available lengths in the problem are said to be the probe radius $R$ and the characteristic polymer correlation length $\xi$, so a scaling law for $D / D_{0}$ depends on the dimensionless ratio $R / \xi$, yielding the prediction

$$
D / D_{0}=\left(\eta_{0} / \eta_{M}\right)+e^{-(R / 5)},
$$

which agrees with the asserted limiting cases $D / D_{0} \rightarrow \eta_{0} / \eta$ as $R \rightarrow \infty$ or $R / \xi \rightarrow \infty$, and $D / D_{0} \approx \psi(R / \xi)$ for $R / \xi$ not too large, if $c$ is large enough that $\eta_{M} / \eta_{0} \gg 1$. In the "semidilute" concentration regime, in which polymer chains overlap, $\xi$ and hence $D / D_{0}$ are predicted to be independent of $M$.

\section{DISCUSSION}

Our experimental data confirm some but not many of the above predictions. For overlapping solutions the scaling law of Eq. (3) was obtained. The $c$ dependence observed in our work agrees with the above models, all of which predicted that $\kappa$ has a power-law dependence on polymer concentration.

The $R$ dependence found experimentally agrees with none of the above models, all of which show a linear dependence of the scaling parameter $\alpha$ on $R$, i.e., the other models predict $\delta=1.0$, while we find $\delta=0$. (The theory of Cukier ${ }^{12}$ is explicitly written for the domain $\kappa R<1$, and may not be applicable to our data.) Furthermore, Eq. (9) was obtained by using the assumption that very large probes do obey the Stokes-Einstein equation. For semidilute polymer solutions, we find that the Stokes-Einstein equation fails, even with very large $(0.62 \mu)$ probe particles and $R / \xi$ as large as 1000 . There is no indication in our data that probe particles would respond to the macroscopic viscosity if only $R$ and $R / \xi$ were made large enough.

The models of Ogston ${ }^{11}$ and of Langevin and Rondelez ${ }^{14}$ both predict $D / D_{0}$ should be independent of $M$ in semidilute polymer solutions, i.e., $\gamma=0$, in disagreement with our experimental value $\gamma=0.8$. With some interpretations ${ }^{14}$ of models of the semidilute regime, the observation $\gamma \neq 0$ is unexpected, even though it has previously been seen experimentally. The experimental data of Laurent and Pietruskiewicz ${ }^{16}$ is consistent with an $M$ dependence of $D / D_{0}$. Langevin and Rondelez ${ }^{14}$ report $\gamma=0$ as an experimental finding. However, these workers were obliged by their technique (centrifugation) to use a substantial probe concentration, and then extrapolate somewhat noisy data to the limit of vanishing probe concentration; their published graphical data are not visibly inconsistent with an $M$ dependence of $D$ / $D_{0}$ in the semidilute regime.

In addition to the specific theoretical models of Sec. III, there is a generalized notion that a polymer solution behaves like a cage or a fishnet. The mobility of a particle through a net depends on the ratio of the particle radius to the mesh size. On identifying the mesh size with the polymer correlation length $\xi$, it is intuitively expected that particles will be mobile if $R / \xi \ll 1$, but will be obstructed if $R / \xi>1$. Our data in the overlapping regime do not support this idea, small particles $(R / \xi<0.5$, e.g., BSA in PEO) being, if anything, more hindered in their motions than are large particles. (In the entangled regime, very large particles are more hindered than smaller particles, but our data on entangled systems is almost all in the range $R / \xi \gg 1$.)

The fallacy in the "fishnet" description of a polymer solution is the implicit assumption that the polymer chains form a background through which the probe moves. Because of hydrodynamic correlations ("interactions"), the polymer chains are subject to nearly the same Brownian forces that the neighboring probe particles are. Thus, a probe particle and its surrounded chains may tend to move as a group, the polymer chains not tending to act as a cage for the probe particle. An analytic evaluation of the caging contribution to $D$ is difficult in a sphere:polymer system. However, Evans and James, ${ }^{17}$ Hanna et al.,${ }^{18}$ Jones and Burfield, ${ }^{19}$ and Marqusee and Deutch ${ }^{20}$ have evaluated $D$ for a sphere in a sphere suspension, using the Mori memory-function formalism to obtain long-term caging effects. If hydrodynamic interactions are neglected, the inhibition of self-diffusion by caging is substantial. If hydrodynamic interactions are added to the calculation, the effect of caging on $D$ is reduced by an order of magnitude or more, and becomes small by comparison with the uncertainty stemming from our lack of exact knowledge of the hydrodynamic interaction tensors.

In summary, we have compared a series of studies of the diffusion of optical probe particles through polymer solutions. Our results had the substantial advantage that our polymer and probe species are chemically distinct, so that the radius of our probe particles and the molecular weight of the polymer could be varied independently. On increasing the polymer concentration beyond the dilute range, our data divide themselves phenomenologically into two categories, which we characterized as the "overlapping" and the "entangled" regimes. The regimes differ most prominently in the degree of failure of the Stokes-Einstein equation which they exhibit, and in the absence or presence of new, "fast" modes in the scattering spectrum. For probe particles in the overlapping regime, a scaling relation connecting $D$ to $c, R$, and $M$ was obtained. The experimental scaling relation is quite different from most theoretical expectations. In the semidilute concentration regime, $D$ is found to depend strongly on $M$ and very weakly on $R$, contrary to predictions that $D$ should depend not at all on $M$, but strongly on $R$.

${ }^{1}$ P. Wiltzius, H. R. Haller, D. S. Cannell, and D. W. Schaeffer, Phys. Rev. Lett. 53, 834 (1984).

${ }^{2}$ G. D. J. Phillies, J. Chem. Phys. 60, 983 (1974).

${ }^{3}$ G. D. J. Phillies, Biopolymers 14, 499 (1975).

${ }^{4}$ K. Ullmann, G. S. Ullmann, and G. D. J. Phillies, J. Colloid. Interface Sci. (in press).

${ }^{5}$ (a) G. S. Ullmann and G. D. J. Phillies, Macromolecules 16, 1947 (1983); (b) G. S. Ullmann, K. Ullmann, R. Lindner, and G. D. J. Phillies, J. Phys. Chem. 89, 692 (1985); (c) G. D. J. Phillies, Biopolymers 24, 379 (1985).

${ }^{6}$ T.-H. Lin and G. D. J. Phillies, J. Phys. Chem. 86, 4073 (1982); J. Colloid 
Interface Sci. 100, 82 (1984); Macromolecules 17, 1686 (1984).

${ }^{7}$ G. D. J. Phillies, J. Phys. Chem. 85, 2838 (1981).

${ }^{8}$ G. D. J. Phillies (in preparation).

${ }^{9}$ C. Ishimoto and T. Tanaka, Phys. Rev. Lett. 39, 474 (1977).

${ }^{10}$ R. N. Haire, W. A. Tisel, J. G. White, and A. Rosenberg, Biopolymers 23 2761 (1984).

${ }^{11}$ A. G. Ogston, P. N. Preston, and J. D. Wells, Proc. R. Soc. London Ser. A 333, $297(1973)$.

${ }^{12}$ R. I. Cukier, Macromolecules 17, 252 (1983).
${ }^{13}$ A. R. Altenberger and M. Tirrell, J. Chem. Phys. 80, 2208 (1984).

${ }^{14} \mathrm{D}$. Langevin and F. Rondelez, Polymer 14, 875 (1978).

${ }^{15}$ P. G. deGennes, Macromolecules 9, 587, 594 (1976).

${ }^{16}$ T. C. Laurent and A. Pietruszkiewicz, Biochim. Biophys. Acta 49, 258 (1974).

${ }^{17}$ G. T. Evans and C. P. James, J. Chem. Phys. 79, 5553 (1983).

${ }^{18} \mathrm{~S}$. Hanna, W. Hess, and R. Klein, Physica A 111, 181 (1982).

${ }^{19}$ R. B. Jones and G. S. Burfield, Physica A 111, $562(1982)$

${ }^{20}$ J. A. Marqusee and J. M. Deutch, J. Chem. Phys. 73, 5396 (1980). 